\title{
Oncologic Outcomes after Radical Cystectomy: Comparison between Primary and Progressive Muscle Invasive Bladder Cancer
}

Khalid Lmezguidi*, Fouad hajji, Abdellatif janane, Mohamed Alami, Ahmed Ameur and Mohamed Abbar

Department of urology, military teaching hospital , Mohamed V university Rabat morocco

\begin{abstract}
Background: Between primary and progressive muscle-invasive bladder cancer, in the current literature, data regarding the prognostic difference and survival between this two entities are controversial.

Objectives: To assess differences in survival between the primary and progressive MIBC and to determine main prognostic factors in muscle-invasive bladder tumors (MIBT).

Material and methods: All patients who were underwent radical cystectomy for MIBC in our institution between 1990 and 2014 were retrospectively evaluated using an institutional database. A total of 308 patients had met inclusion criteria, $218(70,77 \%)$ (Group 1) with primary MIBC and $90(29.22 \%)$ (Group 2) with progressive MIBC. The main variables studied were: age, sex, initial tumor stage of TURs in group 2, pathologic stage (T/N), type of urinary diversion and extent of LND. Survival rate was investigated with Kaplan-Meier method and a multivariate analysis using the Cox regression analysis was performed to evaluate potential prognostic factors.
\end{abstract}

Results: In Group 2, the median time of progression to invasive cancer was 32 months. 2, 3 and 5-year cancer specific survival rate after surgery was $77 \%, 63 \%$ and $51 \%$ in Group 1 and $59 \%, 49 \%$ and $32 \%$ in group 2 , respectively $(p<0.05)$. Analyzing $\mathrm{pN}$ stage, overall 2,3 and 5 -year survival rate were $75 \%, 62 \%$, and $53 \%$ in group 1 and $61 \%, 49 \%$, and $37 \%$ in group 2 respectively for pNO ( $P<0.05)$. On multivariate analysis, lympho-vascular invasion and $\mathrm{pT}$ stage of the primary tumor remained significant independent prognostic factors for cancer-specific survival.

Conclusions: Our study has shown that Progressive MIBC have a worse prognosis than Primary MIBC. Lympho-vascular invasion and Positive nodes in RC specimens seems to be an independent factor that decreases survival in patients with MIBC.

Keywords: Oncologic outcomes; Radical cystectomy; Primary; Progressive; Muscle invasive bladder cancer; Prognosis

\section{Introduction}

Bladder cancer is the second most common genitourinary malignancy, with transitional cell carcinoma (TCC) comprising nearly $90 \%$ of all primary bladder tumors [1]. Muscle-invasive bladder cancer (MIBC) can be classified into two categories: Primary, for those which are muscle-invasive at the time of the diagnosis, and Progressive, for those non-muscle invasive bladder cancers in their earlier stages that will become invasive during their follow-up stages. Approximately $30 \%$ of non-muscle invasive bladder (NMIBT) urothelial tumors progress to muscle-invasive tumors during their follow-up. Radical cystectomy (RC) with bilateral pelvic lymphadenectomy (LA) is the standard of care for muscle-invasive disease, various regimens of preoperative radiation were implemented but failed to demonstrate convincingly any additional benefit $[2,3]$.

Different studies have evaluated the difference between these progressive and primary muscle-invasive tumors in terms of prognosis and survival and results remain controversial. While some studies have found differences in clinical outcomes, others did not.

In the pathologic stage, lymphovascular invasion and lymph node (LN) status have consistently been shown to be the most powerful independent predictors of long-term outcome following radical cystectomy (RC).

The aim of this study was to investigate if there was a difference in the clinical outcomes of patients with progressive and primary muscleinvasive bladder cancers. In addition, we analysed variables such as grade, age, sex, lymphovascular invasion (LVI), pathologic T stage, lymph node status (in the cystectomy specimen), and the detection of metastasis during follow-up (either local or distant) with the aim to bring out independent predictors of cancer-specific survival in muscleinvasive urothelial tumors.

\section{Patients and Methods}

Retrospective data was collected on all 308 patients who underwent RC for bladder urothelial carcinoma at our department from January 1990 to December 2014. Pathologic staging of bladder tumors and LNs was performed per the 2002 TNM classification. All cases treated before 2002 were reclassified. Reclassification per the 2010 TNM classification could not be performed because we had incomplete information on the precise localization of the positive LNs. To assess the maximum tumor stage, the tumor was classified after review of the TURBT and the RC specimens. Histologic grading was performed per the World Health Organization/International Society of Urologic Pathology Classification.

*Corresponding author: Lmezguidi K, Department of urology ,military teaching hospital, Mohamed V university Rabat morocco, Tel: + 22371990282; E-mail: Imezguidikhalid@gmail.com

Received October 18, 2017; Accepted June 20, 2017; Published June 23, 2017

Citation: Lmezguidi K, Hajji F, Janane A, Alami M, Ameur A, et al. (2017) Oncologic Outcomes after Radical Cystectomy: Comparison between Primary and Progressive Muscle Invasive Bladder Cancer. J Cancer Sci Ther 9: 485-489. doi: 10.4172/1948-5956.1000463

Copyright: @ 2017 Lmezguidi K, et al. This is an open-access article distributed under the terms of the Creative Commons Attribution License, which permits unrestricted use, distribution, and reproduction in any medium, provided the original author and source are credited. 
LVI was defined as the presence of tumour cells within an unequivocal endothelium-lined space with no underlying walls of smooth muscle cells, in standard haematoxylin and eosin (H\&E)- stained sections. In selected cases, immunohistochemistry for endothelial cells was done.

To analyze a homogeneous population, all our patients met the following inclusion criteria:

- $\quad$ Primary malignant tumor of the bladder.

- Urothelial carcinoma (UCa).

- No neoadjuvant radiotherapy and/or chemotherapy.

- No adjuvant radiotherapy and/or chemotherapy.

- $\quad$ RC with bilateral PLND

- $\quad$ No positive surgical margins (R1/R2).

Patients undergoing palliative cystectomy and those with upper urinary tract tumors or non-transitional cell carcinoma were excluded.

None of the enrolled patients had LN metastases outside the true pelvis or distant organ metastases in the preoperative assessment or intraoperatively. Our standard preoperative assessment protocol included physical examination, chest $\mathrm{x}$-ray, computed tomography of the pelvis, ultrasonography of the abdomen, bone scan, and excretory urography.

The first group (group 1) included 218 patients with primary muscle-invasive bladder tumors. This group contained patients in whom invasion into the muscular layer was detected at the primary transurethral resection (TUR).

The second group (group 2) was comprised of 90 patients who had intially had a non-muscle-invasive bladder tumor that progressed to muscle-invasive carcinoma during follow-up. The diagnosis of superficial tumor was done by evaluation of the specimen collected after TUR. These patients received additional adjuvant therapy consisting of intravesical instillation with bacille Calmette-Guérin and maintenance therapy or intravesical chemotherapy per their risk stratification. All patients in group 2 underwent cystectomy after Stage T2 disease had been documented pathologically from the specimen collected at the last TUR. Patients with carcinoma in situ or those who had undergone RC for refractory NMIBC were excluded from the study.

All operations, including control cystoscopy, initial transurethral resection, and final radical surgery, were performed at our institution.

Patients with local or distant metastasis detected during follow-up were given chemotherapy. The vinblastine, doxorubicin or epirubicin, and cisplatin regimen were used. After 2002, the gemcitabine and cisplatin regimen were used.

The follow-up strategy after radical surgery consisted of: office visits, serum chemistries, abdominal imaging, and chest radiography every 3-6 months for the first 3 years, with increasing intervals thereafter. Bone scans were ordered when clinically indicated.

Continuous normally distributed variables are presented as the mean (SD), and those not normally distributed as the median (interquartile range). The correlation of the clinical and pathologic variables with survival was investigated by the Cox proportional hazards test. The Kaplan-Meier method was used to derive the cumulative cancerspecific survival (CSS) with the log-rank test used to compare curves of two or more groups. Univariable Cox regression analyses were used to identify differences within pathological variables, and a multivariable
Cox regression analysis to identify prognostic factors. All $P$ values were two-sided and a value of $<0.05$ was considered to indicate significant differences between groups.

\section{Results}

The present study cohort consisted of 308 patients (Table 1); 13 females (4.22\%) and 295 males (95,77\%). Of the 218 patients in group 1 and the 90 in group 2, 10 (3.67) and 5 (5.55) were women, respectively. The mean patient age at surgery was 61.5 years and the mean followup time was 72.6 months for group 1 . The mean age at surgery was 60.3 years and the mean follow-up time was 85.4 months for group 2 $(P>0.05)$. During follow-up, 88 patients died of tumor progression.

The initial tumor stage in the progressive group was pTaG1 in 4 (4.44\%), pTaG2 in 20 (22.22\%), pT1G1 in 4 (4.44\%), pT1G2 in 34 $(37.77 \%)$, and pT1G3 in $28(31.11 \%)$ patients. The median duration between the resection of the first noninvasive tumor and the diagnosis of Stage T2 disease (last TUR before cystectomy) was 32.3 months, ranging from 6 to 178. For pTa tumors, the median duration was 85.6 (ranging from 43 to 190), although it was 27.5 (ranging from to 76 ) months for pT1 tumors $(P<0.05)$.

In group 1, the distribution of cases per the pathologic stage was as follows: 122 (55.96\%) had pT2, 58 (26.60\%) had pT3 and $38(17.43 \%)$ had pT4. In group 2, 50 (55.55\%) had pT2, 24 (26.66\%) had pT3, and 16 (17.77) had pT4.

The 2, 3, and 5-year cancer-specific survival rate was 59\%, 49\%, and $32 \%$ for the patients with progressive tumors and $77 \%, 63 \%$, and $51 \%$ for patients with primary tumors, respectively. All differences in the cancer-specific survival rates were statistically significant between groups 1 and $2,(P<0.05)$. The mean survival time was $61.3 \pm 7.34$ months for group 1 and $89.1 \pm 15.44$ months for group 2 . Using the logrank test, the difference observed in terms of the mean survival time was statistically significant $(P=0.01$; Figure 1$)$.

Of the 90 patients in the progressive group (group 2) and the 218 patients in the primary group (group 1), 73 (81.8\%) and $156(71.55 \%)$ had no positive pelvic lymph nodes after pathologic evaluation (pN0), respectively. For lymph node-negative tumors (pN0), the 2, 3, and 5 -year cancer-specific survival rate was $79 \%, 67 \%$, and $57 \%$ in group 1 and $61 \%, 49 \%$, and $37 \%$ in group 2, respectively. The difference was statistically significant between two groups with the mean survival time $(68.2 \pm 6.44$ months for group 1 and $87 \pm 14.34$ months for group 2; $P$ $=0.034, \log$-rank test) (Figure 2 ).

On multivariate analysis, none of the variables, including sex, age, or tumor grade identified from the cystectomy specimen were significantly associated with patient outcome. Thus, none were believed to be predictive of patient survival. However, lymph node tumor involvement, LVI and the $\mathrm{pT}$ stage of the primary tumor remained significant independent prognostic factors for cancer-specific survival. In addition, the detection of local and/or distant metastasis during follow-up significantly shortened the cancer-specific survival of patients with muscle-invasive bladder cancer (Table 2).

\section{Discussion}

The current study shows a large and clinically significant difference in disease-specific survival between primary and progressive muscleinvasive bladder cancer patients, favouring the primary group. The disease- specific survival is significantly high in the primary group always during follow-up. The 2, 3, and 5-year survival rates are $77 \%$, $63 \%$ and $51 \%$ respectively for patients with a primary invasive tumour 
Citation: Lmezguidi K, Hajji F, Janane A, Alami M, Ameur A, et al. (2017) Oncologic Outcomes after Radical Cystectomy: Comparison between Primary and Progressive Muscle Invasive Bladder Cancer. J Cancer Sci Ther 9: 485-489. doi: 10.4172/1948-5956.1000463

\begin{tabular}{|c|c|c|c|}
\hline Groups of $\mathrm{BCa}$ & Groupe $1 \quad(n=218)$ & Groupe $2(n=90)$ & p-value \\
\hline Age $(y r)$ Mean $\pm S D$ & $61.5 \pm 5.2$ & $69.5 \pm 6.7$ & 0.09 \\
\hline \multicolumn{4}{|c|}{ Gender n (\%) } \\
\hline Male & $210(96.33)$ & $85(94.44)$ & \multirow[t]{2}{*}{0.43} \\
\hline Female & $10(3.67)$ & $5(5.55)$ & \\
\hline Smoking history $\mathrm{n}(\%)$ & $156(71.55)$ & $60(66.66)$ & 0.15 \\
\hline History of pelvis irradiation $\mathrm{n}(\%)$ & $12(5.5)$ & $2(2.22)$ & 0.06 \\
\hline Follow-up duration (months) mean $\pm \mathrm{SD}$ & $72.6 \pm 5.3$ & $85.4 \pm 6.2$ & 0.08 \\
\hline \multicolumn{4}{|c|}{ Initial tumor stage $\mathbf{n}(\%)$} \\
\hline pTaG1 & & $4(4.44)$ & \multirow{5}{*}{--} \\
\hline pTaG2 & & $20(22.22)$ & \\
\hline pT1G1 & & $4(4.44)$ & \\
\hline pT1G2 & & $34(37.77)$ & \\
\hline pT1G3 & & $28(31.11)$ & \\
\hline \multicolumn{4}{|c|}{ Duration between $1^{\text {st }}$ TURBT and stage 2: Median (IQR) } \\
\hline $\mathrm{pTa}$ & -- & $85.6(43-190)$ & \multirow{3}{*}{--} \\
\hline pT1 & -- & $27.5(7-76)$ & \\
\hline Total & -- & $32.3(6-179)$ & \\
\hline \multicolumn{4}{|c|}{ Pathologic stage } \\
\hline pT2 & $122(55.96)$ & $50(55.55)$ & \multirow[b]{3}{*}{0.57} \\
\hline pT3 & $58(26.60)$ & $24(26.66)$ & \\
\hline pT4 & $38(17.43)$ & $16(17.77)$ & \\
\hline \multicolumn{4}{|c|}{ Type of urinary divesion $n(\%)$} \\
\hline Ileal conduit & $86(39.44)$ & $35(38.88)$ & \\
\hline Orthotopic neobladder & $4(1.83)$ & $2(2.22)$ & \\
\hline Continent cutaneous reservoir & $87(39.90)$ & $20(24.44)$ & \\
\hline Data unavailable & $41(18.80)$ & $33(36.66)$ & 0.46 \\
\hline \multicolumn{4}{|c|}{ Extent of LND n (\%) } \\
\hline Standard & $111(50.91)$ & $45(50.00)$ & \multirow{4}{*}{0.77} \\
\hline Extended & $65(29.81)$ & $30(33.33)$ & \\
\hline None & $25(11.46)$ & $11(11.00)$ & \\
\hline Data unavailable & $17(7.79)$ & $4(4.44)$ & \\
\hline
\end{tabular}

Abbreviations: SD=Standard Déviation; IQR=Interquartel Range; TURBT=Transurethral Resection of Bladder Tumor; LND=Lymph-Node Dissection; Bca: Bladder Cancer

Table 1: Patient characteristics.

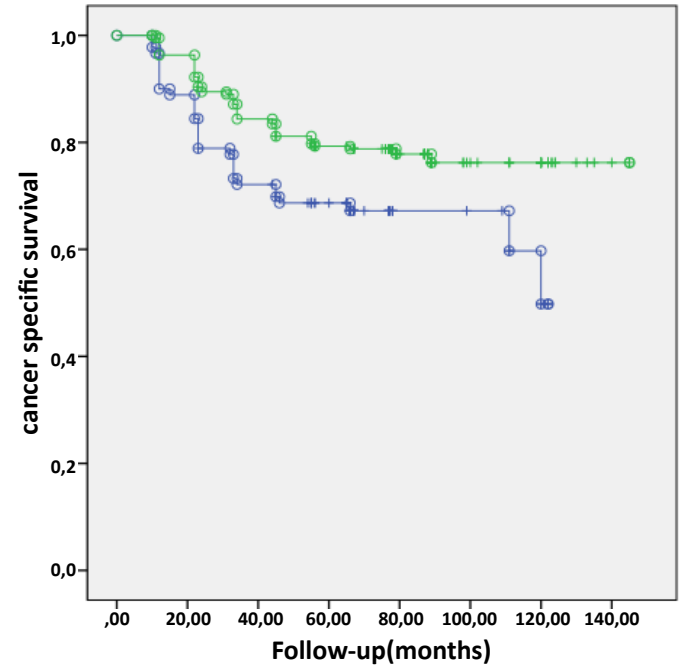

Figure 1 : Kaplan-Meier curve comparing primary and progressive tumors.

and $59 \%, 49 \%$, and $32 \%$ respectively for patients with a progressive invasive tumour. This trend in survival difference between the two study

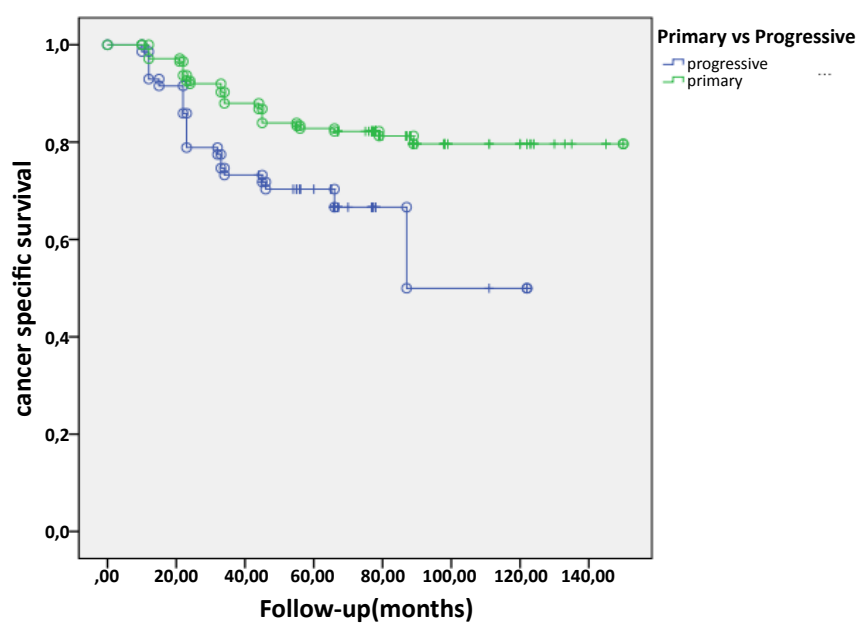

Figure 2 : Kaplan-Meier curve comparing primary and progressive tumors for pNO status.

groups was observed by Schrier, et al. [4] who examinated Nijimen and Rotterdam population showing that the disease- specific survival 


\begin{tabular}{|c|c|c|c|}
\hline Variables & Hazard ratio & P-value & $95 \%$ Confidence Interval \\
\hline Age & 1.09 & 0.13 & $0.88-1.2$ \\
\hline Sex & 0.85 & 0.65 & $0.6-1.8$ \\
\hline Tumor grade & 1.01 & 0.78 & $0.7-1.6$ \\
\hline pT stage & 1.43 & $0.034^{\circ}$ & $1.1-1.5$ \\
\hline $\begin{array}{l}\text { LN status } \\
(\mathrm{pN}+)\end{array}$ & 1.56 & $0.04^{\circ}$ & $1.2-2.5$ \\
\hline Metastasis during follow-up & 8.45 & $0.001^{\circ}$ & $5.4-16.2$ \\
\hline LVI & 2.8 & $0.003^{\circ}$ & $1.2-5.1$ \\
\hline
\end{tabular}

Table 2: Multivariable analysis results.

appears to be approximately twice as high in the primary group. The 3 and 5 -year survival rates are $67 \%$ and $55 \%$ respectively for patients with a primary invasive tumour and $37 \%$ and $28 \%$ respectively for patients with a progressive invasive tumour. Vaidya et al. [5] found a 2-year survival rate of $49 \%$ for those with primary (de novo) invasive tumors and $79 \%$ for those with progression from less than $\mathrm{T} 2$ at presentation concluding that progressive tumors had a worse prognosis than initially muscle-invasive tumors. Like this finding, Parra-Lopez, et al. [6] retrospectively reviewed the records of patients undergoing radical cystectomy. Their overall survival rate after 1 and 3 years of surgery was $86.9 \%$ and $70.2 \%$ for primary muscle-invasive tumors and $75.7 \%$ and $32.4 \%$ for progressive tumors. While these studies show significant differences between two study groups, Soloway [7] argued regarding the presence of difference. Türkölmez [7] examinating oncological outcomes after surgery between two groups, they found the 2,3 , and 5 -year cancer-specific survival rate was $72 \%, 61 \%$, and $43 \%$ for patients with progressive tumors and $75 \%, 62 \%$, and $54 \%$ for patients with primary tumors, respectively and no statistically significance exists between these results. They conclude that patients with progressive muscle-invasive urothelial tumors do not have a worse prognosis than do those with primary tumors.

Recent data published by Moschini et al. [8] with a large sample of 768 consecutive patients treated with radical cystectomy following primary or progressive MIBC, they found That The 10-year RFS, CSM and OM rates for primary vs. progressive status were 43 vs. $36 \%$ ( $\mathrm{P}=0.01), 43$ vs. $37 \%(\mathrm{P}=0.01)$, and 35 vs. $28 \%(\mathrm{P}=0.03)$, respectively. They suggested that progressive status was associated with a higher $\mathrm{CSM}, \mathrm{OM}$ and recurrence rate after RC

When we excluded the patients with positive lymph nodes in the cystectomy specimen and re-evaluated the outcomes statistically, similar Kaplan-Meier curves were reached with the 2, 3, and 5-year cancer-specific survival rate being $79 \%, 67 \%$, and $57 \%$ in group 1 and $61 \%, 49 \%$, and $37 \%$ in group 2 , respectively. The same results are shown in the study of Lopez, et al. [6] after analyzing pN stage, the overall 1 -year and 3 -year survival rate were $90.7 \%$ and $64.3 \%$ for $\mathrm{pN}(+)$ tumors and $77.7 \%$ and $48.2 \%$ respectively for $\mathrm{pN}(-)$.

The mechanism behind this observed difference is not so easily understood. Since survival figures of the primary invasive patients is comparable with the literature, the explanation for the significant differences between the two groups must be found in the worse survival of patients with progressive muscle-invasive bladder cancer. One possible explanation could be that in high risk superficial bladder tumours both therapy-sensitive and -insensitive cells coexist. Intravesical therapy given to patients with high-risk superficial bladder cancer might select for resistant clones, and the more aggressive tumor cells might continue to grow and lead to the development of a progressive tumor.
Another possible explanation could be a finding published by ElAbbady, et al. [9]. They compared 16 patients with progressive invasive tumours with 20 patients who were diagnosed with primary invasive tumours, all undergoing cystectomy. On meticulous histopathological examination, they found that patients who underwent previous transurethral resections had significantly more local spread of malignant cells into the bladder muscle as compared to patients with primary invasive tumours. Since they could demonstrate that intravesical pressure reaches as high as $80 \mathrm{~cm}$ water, they suggested some malignant cells penetrated through the denuded urothelium during resection because of high intravesical pressures. Similarly, random biopsies during resection of superficial tumours might cause tumour cell implantation at the site of the damaged mucosa, and influence the prognosis. However, two large series clearly demonstrated that the risk of recurrence and the risk of progression is almost the same comparing a "biopsy policy" with a "no biopsy policy" [10,11].

It has been previously reported that the pathological stage and the nodal status are the most important prognostic factors for patients undergoing RC for bladder cancer [12-14]. In the present study, as would be expected, pT stage was an idependant prognostic factor of survival after RC as well as lymph node status. The appearance of local and/or distant metastasis during follow-up dramatically shortened the life of patients with cystectomized bladder cancer in our study population (hazards ratio 8.45 ).

On multivariable analysis, our study shows an idependent prognostic value of lymphovascular invasion. In a multicentre study Bolenz, et al. [15] identified the presence of LVI in the surgical specimen as an independent predictor of survival in patients with node-negative UBC treated with RC and pelvic LA. They identified LVI in $26.8 \%$ of patients with node-negative disease, which is in line with the proportion reported in other studies, specifically the studies of Lotan et al \& Harrada, et al. $[16,17]$. LVI occurred more frequently in tumours with higher stage and grade, but also $\beta \approx 30 \%$ of pT1 tumours showed LVI, indicating metastatic potential despite a low stage. The same results are shown in other large previous studies [17-20]. Controversially, Bassi, et al. [21] and Hara, et al. [22] established that LVI was not a predictor of survival.

The process of LVI remains poorly understood but there is a consensus that it represents an early step in the systemic spread of malignant cells $[22,23]$. LVI can be regarded as a surrogate marker for the presence of lymphatic micrometastases at the time of RC. In a large multicentre study, Lotan, et al. [16] previously evaluated 151 LVI-positive patients with node-negative UBC after RC and showed that LVI is a predictor of recurrence, CSS, and OS. In their study, the prevalence of LVI increased with higher pathological stage and grade. The presence of LVI retained independent prognostic value in 
Citation: Lmezguidi K, Hajji F, Janane A, Alami M, Ameur A, et al. (2017) Oncologic Outcomes after Radical Cystectomy: Comparison between Primary and Progressive Muscle Invasive Bladder Cancer. J Cancer Sci Ther 9: 485-489. doi: 10.4172/1948-5956.1000463

competing- risks regression models in which other-because mortality was considered as a competing risk. Given these findings and some confirmatory retrospective studies $[16,20,23]$, LVI has been suggested to be included in clinical staging models of UBC.

\section{Conclusion}

Our study has shown that patients with progressive muscle-invasive urothelial tumors have a worse prognosis than those with primary tumors. Therefore, in high risk patients with recurrent or persisting tumours at initial evaluation, radical surgery should be seriously considered. For both groups, pT stage, LN status and LVI seem to be independent predictors of decreased cancer-specific survival.

\section{References}

1. Jemal A, Siegel R, Ward E, Hao Y, Xu J, et al. (2009) Cancer statistics. CA Cancer J Clin 59: 225-249.

2. Stenzl A, Cowan NC, De Santis M, Kuczyk MA, Merseburger AS, et al. (2011) Treatment of muscle- invasive and metastatic bladder cancer: Update of the EAU guidelines. Eur Urol 59: 1009-1018.

3. Stein JP, Lieskovsky G, Cote R, Groshen S, Feng AC, et al. (2001) Radical cystectomy in the treatment of invasive bladder cancer: long-term results in 1054 patients. J Clin Oncol 19: 666-675.

4. Schrier BP, Hollander MP, Van Rhijn BW, Kiemeney LA, Witjes JA, et al. (2004) Prognosis of muscle-invasive bladder cancer: Difference between primary and progressive tumours and implications for therapy. Eur Urol 45: 292-296.

5. Vaidya A, Soloway MS, Hawke C, Tiguert R, Civantos F (2001) De novo muscle invasive bladder cancer: Is there a change in trend? J Urol 165: 47-50.

6. Parra-Lopez L, Villegas-Osorio JF, Congregado Ruiz CB, Osman-García I, Medina López RA, et al. (2014) P121 Prognostic factors and survival differences between primary and progressive muscle-invasive bladder cancer. Eur Urol Supplements 13: 103-194.

7. Türkölmez K, Tokgöz H, Reşorlu B, Köse K, Bedük Y (2007) Muscle-invasive bladder cancer: Predictive factors and prognostic difference between primary and progressive tumors. Urology 70: 477-481.

8. Moschini M, Sharma V, Dell'oglio P, Cucchiara V, Gandaglia G, et al. (2016) Comparing long-term outcomes of primary and progressive carcinoma invading bladder muscle after radical cystectomy. BJU Int 117: 604-610.

9. El-Abbady AA, Shoukry MS, Hanno AG, Younis LK, Abdel-Rahman M (2002) Repeated transurethral resection of recurrent superficial bladder tumors: Does it affect the spread and stage of the tumor? Scand J Urol Nephrol 36: 60-64.

10. Kiemeney LALM, Witjes JA, Heijbroek RP, Koper NP, Verbeek AL, et al
(1994) Should random urothelial biopsies be taken from patients with primary superficial bladder cancer? A decision analysis. Br J Urol 73: 164-171.

11. Van der Meijden A, Oosterlinck W, Brausi M, Kurth KH, Sylvester R, et al. (1999) Significance of bladder biopsies in Ta, T1 bladder tumors: A report from the EORTC genito-urinary tract cancer cooperative group. EORTC-GU Group Superficial Bladder Committee. Eur Urol 35: 267-271.

12. Shariat SF, Karakiewicz PI, Palapattu GS, Lotan Y, Rogers CG, et al. (2006) Outcomes of radical cystectomy for transitional cell carcinoma of the bladder: A contemporary series from the bladder cancer research consortium. J Urol 176: 2414-2422.

13. Margulis V, Lotan Y, Montorsi F, Shariat SF (2008) Predicting survival after radical cystectomy for bladder cancer. BJU Int 102: 15-22.

14. Bolenz C, Herrmann E, Bastian PJ, Michel MS, Wülfing C, et al (2010) Lymphovascular invasion is an independent predictor of oncological outcomes in patients with lymph node-negative urothelial bladder cancer treated by radical cystectomy: A multicentre validation trial. BJU Int 106: 493-499

15. Lotan Y, Gupta A, Shariat SF, Palapattu GS, Vazina A, et al. (2005) Lymphovascular invasion is independently associated with overall survival cause-specific survival, and local and distant recurrence in patients with negative lymph nodes at radical cystectomy. J Clin Oncol 23: 6533-6539.

16. Harada K, Sakai I, Hara I, Eto H, Miyake H (2005) Prognostic significance of vascular invasion in patients with bladder cancer who underwent radical cystectomy. Int J Urol 12: 250-255.

17. Horikawa Y, Kumazawa T, Narita S, Inoue T, Yuasa T, et al. (2007) Lymphatic invasion is a prognostic factor for bladder cancer treated with radical cystectomy. Int J Clin Oncol 12: 131-136.

18. Hara S, Miyake H, Fujisawa M, Okada H, Arakawa S, et al. (2001) Prognostic variables in patients who have undergone radical cystectomy for transitional cell carcinoma of the bladder. Jpn J Clin Oncol 31: 399-402.

19. Leissner J, Koeppen C, Wolf HK (2003) Prognostic significance of vascular and perineural invasion in urothelial bladder cancer treated with radical cystectomy. J Urol 169: 955-960.

20. Bassi P, Ferrante GD, Piazza N, Spinadin R, Carando R, et al. (1999) Prognostic factors of outcome after radical cystectomy for bladder cancer: A retrospective study of a homogeneous patient cohort. J Urol 161: 1494-1497.

21. Karpanen T, Alitalo K (2008) Molecular biology and pathology of lymphangiogenesis. Annu Rev Pathol 3: 367-397.

22. Padera TP, Kadambi A, Di Tomaso E, Carreira CM, Brown EB, et al. (2002) Lymphatic metastasis in the absence of functional intratumor lymphatics. Science 296: 1883-1886.

23. Streeper NM, Simons CM, Konety BR, Muirhead DM, Williams RD, et al. (2009) The significance of lymphovascular invasion in transurethral resection? of bladder tumour and cystectomy specimens on the survival of patients with urothelial bladder cancer. BJU Int 103: 475-479. 